\title{
Characterization for the Convergence of Krasnoselskij Iteration for Non-Lipschitzian Operators
}

\author{
Ştefan M. Şoltuz ${ }^{1,2}$ and B. E. Rhoades ${ }^{3}$ \\ ${ }^{1}$ Departamento de Matematicas, Universidad de Los Andes, Carrera 1 no. 18A-10, Bogota, Colombia \\ 2 "Tiberiu Popoviciu” Institute of Numerical Analysis, 400110 Cluj-Napoca, Romania \\ ${ }^{3}$ Department of Mathematics, Indiana University, Bloomington, IN 47405-7106, USA
}

Correspondence should be addressed to Ştefan M. Şoltuz, smsoltuz@gmail.com

Received 13 August 2007; Revised 2 February 2008; Accepted 24 February 2008

Recommended by Enrico Obrecht

We establish the convergence of Krasnoselskij iteration for various classes of non-Lipschitzian operators.

Copyright (C) 2008 Ş. M. Şoltuz and B. E. Rhoades. This is an open access article distributed under the Creative Commons Attribution License, which permits unrestricted use, distribution, and reproduction in any medium, provided the original work is properly cited.

\section{Introduction}

Let $X$ be a real Banach space; $B$ a nonempty, convex subset of $X$; and $T: B \rightarrow B$ an operator. Let $x_{0} \in B$. The following iteration is known as Krasnoselskij iteration (see [1]):

$$
x_{n+1}=(1-\lambda) x_{n}+\lambda T x_{n} .
$$

The map $J: X \rightarrow 2^{X^{*}}$ given by $J x:=\left\{f \in X^{*}:\langle x, f\rangle=\|x\|^{2},\|f\|=\|x\|\right\}$, for all $x \in X$, is called the normalized duality mapping. It is easy to see that we have

$$
\langle y, j(x)\rangle \leq\|x\|\|y\|, \quad \forall x, y \in X, \forall j(x) \in J(x) .
$$

Denote

$\Psi:=\{\psi \mid \psi:[0,+\infty) \longrightarrow[0,+\infty)$ is astrictly increasing map with $\psi(0)=0\}$.

Definition 1.1. Let $X$ be a real Banach space, and let $B$ be a nonempty subset of $X$. A map $T$ : $B \rightarrow B$ is called uniformly pseudocontractive if there exists a map $\psi \in \Psi$ and $j(x-y) \in J(x-y)$ such that

$$
\langle T x-T y, j(x-y)\rangle \leq\|x-y\|^{2}-\psi(\|x-y\|), \quad \forall x, y \in B .
$$


A map $S: X \rightarrow X$ is called uniformly accretive if there exists a map $\psi \in \Psi$ and $j(x-y) \in$ $J(x-y)$ such that

$$
\langle S x-S y, j(x-y)\rangle \geq \psi(\|x-y\|), \quad \forall x, y \in X
$$

Taking $\psi(a):=\psi(a) \cdot a$, for all $a \in[0,+\infty),(\psi \in \Psi)$, reduces to the usual definitions of $\psi$-strongly pseudocontractive and $\psi$-strongly accretive. Taking $\psi(a):=\gamma \cdot a^{2}, \gamma \in(0,1)$, for all $a \in[0,+\infty),(\psi \in \Psi)$, we get the usual definitions of strongly pseudocontractive and strongly accretive. Therefore, the class of strongly pseudocontractive maps is included stricly in the class of $\psi$-strongly pseudocontractive maps. The example from [2] shows that this inclusion is proper. Remark, further, that the class of $\psi$-strongly pseudocontractive maps is also included strictly in the class of uniformly pseudocontractive maps (see also [3]).

We will give a characterization for the convergence of (1.1) when applied to uniformly pseudocontractive operators. For this purpose, we need the following lemma similar to [4, Lemma 1]. Next, $\mathbb{N}$ denotes the set of all natural numbers.

Lemma 1.2. Let $\left\{a_{n}\right\}$ be a positive bounded sequence and assume that there exists $n_{0} \in \mathbb{N}$ such that

$$
a_{n+1} \leq(1-\lambda) a_{n}+\lambda a_{n+1}-\lambda \frac{\psi\left(a_{n+1}\right)}{a_{n+1}}+\lambda \varepsilon_{n}, \quad \forall n \geq n_{0},
$$

where $\lambda \in(0,1), \varepsilon_{n} \geq 0$, for all $n \in \mathbb{N}$ and $\lim _{n \rightarrow \infty} \varepsilon_{n}=0$. Then $\lim _{n \rightarrow \infty} a_{n}=0$.

Proof. There exists an $M>0$ such that $a_{n} \leq M$, for all $n \in \mathbb{N}$. Denote $a:=\lim \inf a_{n}$. We will prove that $a=0$. Suppose on the contrary that $a>0$. Then there exists an $N_{1} \in \mathbb{N}$ such that

$$
a_{n} \geq \frac{a}{2}, \quad \forall n \geq N_{1}
$$

From $\lim _{n \rightarrow \infty} \varepsilon_{n}=0$, we know that there exists an $N_{2} \in \mathbb{N}$ such that

$$
\varepsilon_{n} \leq \frac{\psi(a / 2)}{2 M}, \quad \forall n \geq N_{2} .
$$

Set $N_{0}:=\max \left\{N_{1}, N_{2}\right\}$. Using the fact that $-(1 / M) \geq-\left(1 / a_{n+1}\right)$,we get the following:

$$
\begin{aligned}
a_{n+1} & \leq(1-\lambda) a_{n}+\lambda a_{n+1}-\lambda \frac{\psi\left(a_{n+1}\right)}{a_{n+1}}+\lambda \varepsilon_{n} \\
& \leq(1-\lambda) a_{n}+\lambda a_{n+1}-\lambda \frac{\psi(a / 2)}{M}+\lambda \frac{\psi(a / 2)}{2 M} \\
& \leq(1-\lambda) a_{n}+\lambda a_{n+1}-\lambda \frac{\psi(a / 2)}{2 M}
\end{aligned}
$$

which implies that $(1-\lambda) a_{n+1} \leq(1-\lambda) a_{n}-\lambda((\psi(a / 2)) / 2 M)$, or

$$
a_{n+1} \leq a_{n}-\frac{\lambda}{1-\lambda} \frac{\psi(a / 2)}{2 M} \leq a_{n}-\lambda \frac{\psi(a / 2)}{2 M},
$$


since $-(\lambda /(1-\lambda)) \leq-\lambda$. Thus $\lambda(\psi(a / 2)) / 2 M \leq a_{n}-a_{n+1}$, which implies that $\sum \lambda<\infty$, in contradiction to $\sum \lambda=\infty$. Therefore, $\lim \inf a_{n}=0$. Hence there exists a subsequence $\left\{a_{n_{j}}\right\} \subset$ $\left\{a_{n}\right\}$ such that $\lim _{j \rightarrow \infty} a_{n_{j}}=0$. Fix $\varepsilon>0$. Then there exists an $n_{3} \in \mathbb{N}$ such that

$$
a_{n_{j}}<\frac{\varepsilon}{4}, \quad \forall j \geq n_{3} .
$$

Also there exists an $n_{4} \in \mathbb{N}$ such that

$$
\varepsilon_{n}<\frac{\psi(\varepsilon / 4)}{2 M}, \quad \forall n \geq n_{4}
$$

Define $n_{0}:=\max \left\{n_{3}, n_{4}, N_{0}\right\}$. We claim that $a_{n_{j}+k}<\varepsilon / 4$ for each $j>n_{0}$ and each $k>0$. Suppose not. Then there exists an $n_{0}$ and a $k>0$ such that

$$
a_{n_{j}+k} \geq \frac{\varepsilon}{4}
$$

For this $n_{j}$, let $k$ denote the smallest positive integer for which (1.13) is true. Then $a_{n_{j}+k-1} \leq \varepsilon / 4$.

From (1.6),

$$
\begin{aligned}
a_{n_{j}+k} & \leq(1-\lambda) a_{n_{j}+k-1}+\lambda a_{n_{j}+k}-\lambda \frac{\psi\left(a_{n_{j}+k}\right)}{a_{n_{j}+k}}+\lambda \varepsilon_{n_{j}+k-1} \\
& \leq(1-\lambda) a_{n_{j}+k-1}+\lambda a_{n_{j}+k}-\frac{\lambda \psi(\varepsilon / 4)}{a_{n_{j}+k}}+\lambda \frac{\psi(\varepsilon / 4)}{2 M} \\
& \leq(1-\lambda) a_{n_{j}+k-1}+\lambda a_{n_{j}+k}-\lambda \frac{\psi(\varepsilon / 4)}{2 M}
\end{aligned}
$$

which implies that $a_{n_{j}+k} \leq(\varepsilon / 4)-(\lambda /(1-\lambda))(\psi(\varepsilon / 4) / 2 M)$. This leads to the contradiction:

$$
\frac{\varepsilon}{4} \leq a_{n_{j}+k} \leq \frac{\varepsilon}{4}-\frac{\lambda}{1-\lambda} \frac{\psi(\varepsilon / 4)}{2 M}<\frac{\varepsilon}{4}
$$

Therefore, $a_{n_{j}+k}<\varepsilon / 4$, for all $k \in \mathbb{N}$, and each $j>n_{0}$, hence $\lim _{n \rightarrow \infty} a_{n}=0$.

\section{Main result}

Theorem 2.1. Let $X$ be a real Banach space, $B$ a nonempty, closed, convex, bounded subset of $X$. Let $T$ : $B \rightarrow B$ be a uniformly pseudocontractive and uniformly continuous operator with $F(T) \neq \varnothing$. Then for $x_{0} \in B$, the Krasnoselskij iteration (1.1) converges to the fixed point of $T$ if and only if $\lim _{n \rightarrow \infty} \| x_{n+1}-$ $x_{n} \|=0$.

Proof. Since $T$ is a self-map of $B$, which is bounded and convex, then, from (1.1), each $x_{n} \in B$, so $\left\{x_{n}\right\}$ is bounded for each $n \in \mathbb{N}$. Uniqueness of the fixed point follows from (1.4). If $\left\{x_{n}\right\}$ converges to the fixed point of $T$, that is, $\lim _{n \rightarrow \infty} x_{n}=x^{*}$, then, obviously, $\lim _{n \rightarrow \infty}\left\|x_{n+1}-x_{n}\right\|=0$. Conversely, we will prove that if $\lim _{n \rightarrow \infty}\left\|x_{n+1}-x_{n}\right\|=0$, then $\lim _{n \rightarrow \infty} x_{n}=x^{*}$. Suppose that 
$x_{n}=x^{*}$ for some $n \in \mathbb{N}$. Then from (1.1), it follows that $x_{m}=x^{*}$ for each $m>n$, and the theorem is proved. Now suppose that $x_{n} \neq x^{*}$ for each $n \in \mathbb{N}$. Using (1.1) and (1.2),

$$
\begin{aligned}
& \left\|x_{n+1}-x^{*}\right\|^{2} \\
& \quad=\left\langle x_{n+1}-x^{*}, j\left(x_{n+1}-x^{*}\right)\right\rangle \\
& \quad=\left\langle(1-\lambda)\left(x_{n}-x^{*}\right)+\lambda\left(T x_{n}-T x^{*}\right), j\left(x_{n+1}-x^{*}\right)\right\rangle \\
& \quad=(1-\lambda)\left\langle\left(x_{n}-x^{*}\right), j\left(x_{n+1}-x^{*}\right)\right\rangle+\lambda\left\langle T x_{n}-T x^{*}, j\left(x_{n+1}-x^{*}\right)\right\rangle \\
& \quad \leq(1-\lambda)\left\|x_{n}-x^{*}\right\|\left\|x_{n+1}-x^{*}\right\|+\lambda\left\langle T x_{n+1}-T x^{*}, j\left(x_{n+1}-x^{*}\right)\right\rangle+\lambda\left\langle T x_{n}-T x_{n+1}, j\left(x_{n+1}-x^{*}\right)\right\rangle \\
& \quad \leq(1-\lambda)\left\|x_{n}-x^{*}\right\|\left\|x_{n+1}-x^{*}\right\|+\lambda\left\|x_{n+1}-x^{*}\right\|^{2}-\lambda \psi\left(\left\|x_{n+1}-x^{*}\right\|\right)+\lambda\left\|T x_{n}-T x_{n+1}\right\|\left\|x_{n+1}-x^{*}\right\| \\
& \quad \leq\left\|x_{n+1}-x^{*}\right\|\left((1-\lambda)\left\|x_{n}-x^{*}\right\|+\lambda\left\|x_{n+1}-x^{*}\right\|-\lambda \frac{\psi\left(\left\|x_{n+1}-x^{*}\right\|\right)}{\left\|x_{n+1}-x^{*}\right\|}+\lambda\left\|T x_{n}-T x_{n+1}\right\|\right) .
\end{aligned}
$$

Hence

$$
\left\|x_{n+1}-x^{*}\right\| \leq(1-\lambda)\left\|x_{n}-x^{*}\right\|+\lambda\left\|x_{n+1}-x^{*}\right\|-\lambda \frac{\psi\left(\left\|x_{n+1}-x^{*}\right\|\right)}{\left\|x_{n+1}-x^{*}\right\|}+\lambda\left\|T x_{n}-T x_{n+1}\right\| .
$$

Since $\lim _{n \rightarrow \infty}\left\|x_{n+1}-x_{n}\right\|=0$ and $T$ is uniformly continuous, it follows that

$$
\lim _{n \rightarrow \infty}\left\|T x_{n}-T x_{n+1}\right\|=0
$$

Set $a_{n}=\left\|x_{n}-x^{*}\right\|, \varepsilon_{n}=\left\|T x_{n}-T x_{n+1}\right\|$ and use Lemma 1.2 to obtain the conlcusion.

Remark 2.2. (1) If $B$ is not bounded, then Theorem 2.1 holds under the assumption that $\left\{x_{n}\right\}$ is bounded.

(2) If $T(B)$ is bounded, then $\left\{x_{n}\right\}$ is bounded.

(3) If $T$ is strongly pseudocontractive, then automatically $F(T) \neq \varnothing$.

\section{Further results}

Let $I$ denote the identity map. A map $T: B \rightarrow B$ is called pseudocontractive if there exists $j(x-y) \in J(x-y)$ such that $\langle T x-T y, j(x-y)\rangle \leq\|x-y\|^{2}$.

Remark 3.1. The operator $T$ is a (uniformly, strongly) pseudocontractive map if and only if $(I-T)$ is a (uniformly, strongly) accretive map.

Remark 3.2. (1) Let $T, S: X \rightarrow X$, and let $f \in X$ be given. A fixed point for the map $T x=$ $f+(I-S) x$, for all $x \in X$, is a solution for $S x=f$.

(2) Let $f \in X$ be a given point. If $S$ is an accretive map, then $T=f-S$ is a strongly pseudocontractive map. 
Consider Krasnoselskij iteration with $T x=f+(I-S) x$,

$$
x_{n+1}=(1-\lambda) x_{n}+\lambda\left(f+(I-S) x_{n}\right) .
$$

Remarks 3.1 and 3.2 and Theorem 2.1 lead to the following result.

Corollary 3.3. Let $X$ be a real Banach space and let $S: X \rightarrow X$ be a uniformly accretive and uniformly continuous operator, with $(I-S)(X)$ bounded. Suppose that $S x=f$ has a solution. Then for any $x_{0} \in$ $X$, the Krasnoselskij iteration (3.1) converges to the solution of $S x=f$ if and only if $\lim _{n \rightarrow \infty} \| x_{n+1}-$ $x_{n} \|=0$.

Let $S$ be an accretive operator. The operator $T x=f-S x$ is strongly pseudocontractive for a given $f \in X$. A solution for $T x=x$ becomes a solution for $x+S x=f$. Consider Krasnoselskij iteration with $T x:=f-S x$,

$$
x_{n+1}=(1-\lambda) x_{n}+\lambda\left(f-S x_{n}\right) .
$$

Again, using Remarks 3.1 and 3.2 and Theorem 2.1, we obtain the following result.

Corollary 3.4. Let $X$ be a real Banach space and let $S: X \rightarrow X$ be an accretive and uniformly continuous operator, with $(I-S)(X)$ bounded. Suppose that $x+S x=f$ has a solution. Then for $x_{0} \in X$, the Krasnoselskij iteration (3.2) converges to the solution of $x+S x=f$ if and only if $\lim _{n \rightarrow \infty}\left\|x_{n+1}-x_{n}\right\|=0$.

Remark 3.5. If (1.4) holds for all $x \in B$ and $y:=x^{*} \in F(T)$, then such a map is called uniformly hemicontractive. It is trivial to see that our results hold for the uniformly hemicontractive maps.

\section{Acknowledgment}

The authors are indebted to referee for carefully reading the paper and for making useful suggestions.

\section{References}

[1] M. A. Krasnosel'skii, "Two remarks on the method of successive approximations," Uspekhi Matematicheskikh Nauk, vol. 10, no. 1, pp. 123-127, 1955.

[2] M. O. Osilike, "Iterative solution of nonlinear equations of the $\phi$-strongly accretive type," Journal of Mathematical Analysis and Applications, vol. 200, no. 2, pp. 259-271, 1996.

[3] C. E. Chidume and C. O. Chidume, "Convergence theorems for fixed points of uniformly continuous generalized $\phi$-hemi-contractive mappings," Journal of Mathematical Analysis and Applications, vol. 303, no. 2, pp. 545-554, 2005.

[4] Ş. M. Şoltuz, "New technique for proving the equivalence of Mann and Ishikawa iterations," Revue d'Analyse Numérique et de Théorie de l'Approximation, vol. 34, no. 1, pp. 103-108, 2005. 


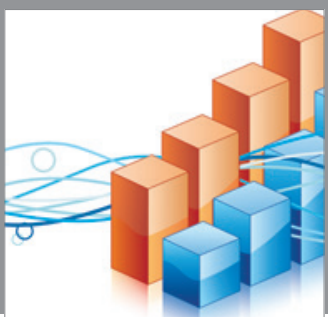

Advances in

Operations Research

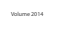

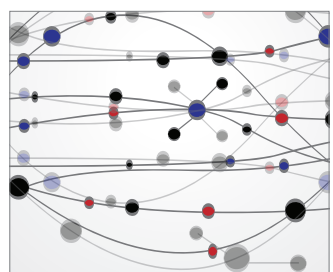

\section{The Scientific} World Journal
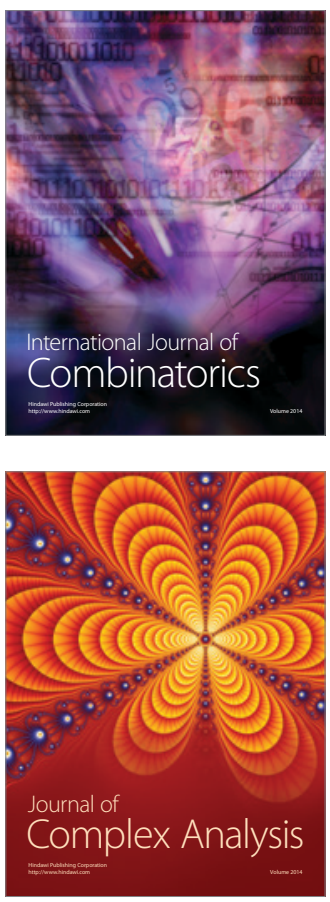

International Journal of

Mathematics and

Mathematical

Sciences
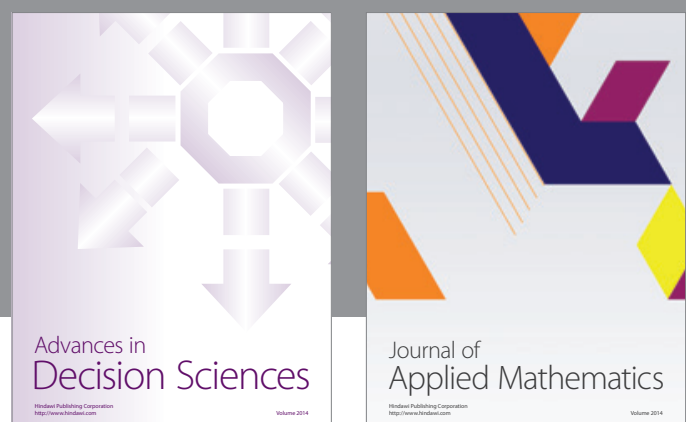

Journal of

Applied Mathematics
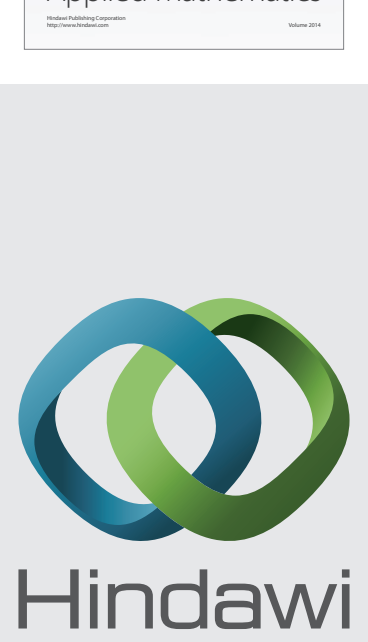

Submit your manuscripts at http://www.hindawi.com
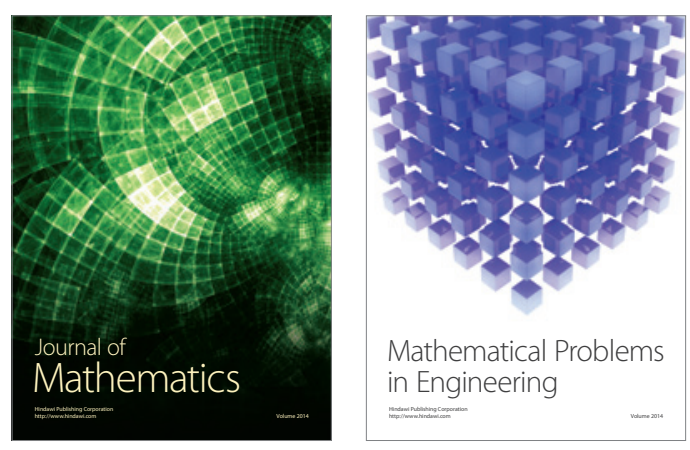

Mathematical Problems in Engineering
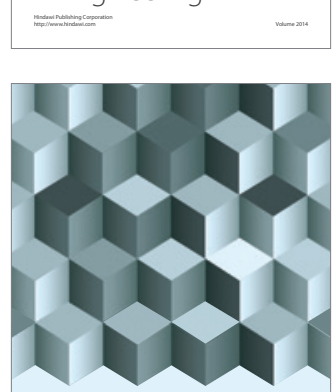

Journal of

Function Spaces
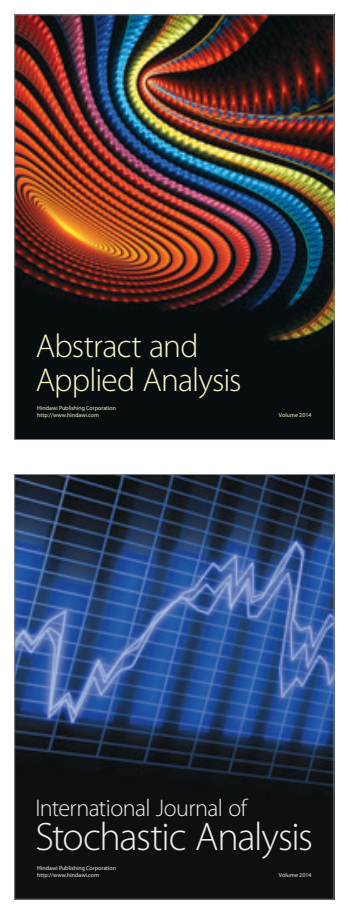

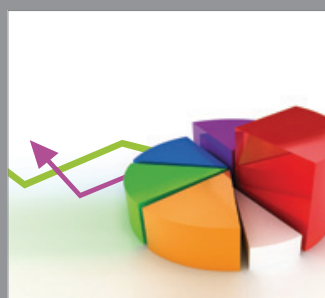

ournal of

Probability and Statistics

Promensencen
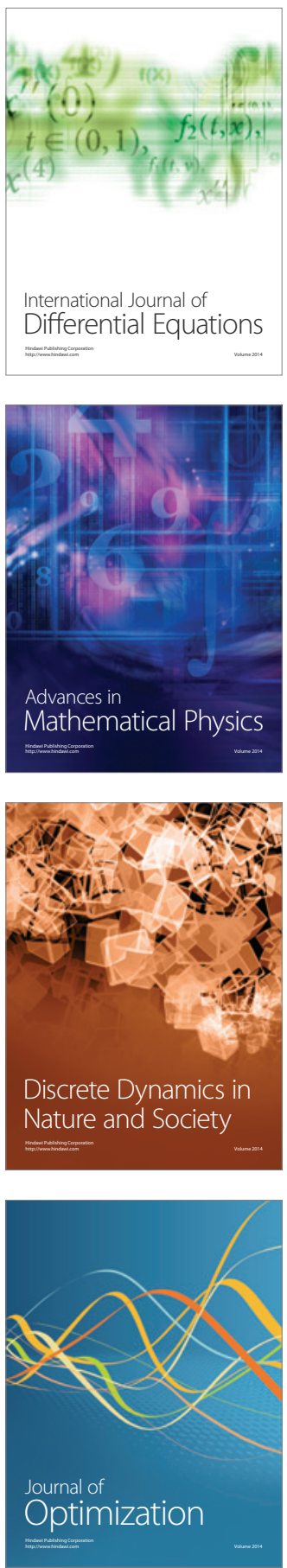